\title{
PERSPECTIVA DEL DERECHO DEL MEDIO AMBIENTE Y DE LAS POLÍTICAS AMBIENTALES DE LA UNIÓN EUROPEA
}

\author{
JORGE AGUDO GONZÁLEZ \\ Profesor titular de Derecho Administrativo \\ Universidad Autónoma de Madrid \\ LORENA TRUJILLO PARRA \\ Becaria de investigación \\ Universidad Autónoma de Madrid
}


Sumario: 1. Introducción. 2. Actividad de las instituciones en el ámbito de la política europea de medio ambiente. 2.1. Actos conjuntos del Consejo y del Parlamento Europeo. 2.2. Actos del Consejo. 2.3. Actos de la Comisión. 2.4. Actos del Parlamento. 2.5. Otros.

\section{Introducción}

En el período de tiempo transcurrido desde finales del primer trimestre de 2012 hasta mediados del mes de octubre de este mismo año, la Unión Europea ha llevado a cabo una intensa actividad en el marco de su política medioambiental. Como es habitual, gozan de mayor protagonismo, al menos desde una perspectiva puramente cuantitativa, los actos jurídicos dictados en ejecución y desarrollo de normas aprobadas con anterioridad, así como los actos jurídicos no vinculantes. Aunque son varias las materias objeto de la acción comunitaria a lo largo de este período, en esta crónica, si alguna materia destaca sobre el resto, esta es la protección de especies piscícolas. La exposición se ordena en función de la institución emisora del acto jurídico, y dentro de la actividad de cada institución, en la medida de lo posible se ordena temáticamente.

\section{Actividad de las instituciones en el ámbito de la política europea de medio ambiente}

\subsection{Actos conjuntos del Consejo y del Parlamento Europeo}

Tres son los actos jurídicos vinculantes que deben ser destacados. En primer lugar, el Reglamento 528/2012, de 22 de mayo de 2012, relativo a la comercialización y el uso de los biocidas. Este reglamento modifica la Directiva 98/8/CE del Parlamento Europeo y del Consejo, que estableció las normas sobre la comercialización de biocidas dentro de la Comunidad.

El objetivo principal del Reglamento es mejorar la libre circulación de biocidas dentro de la Unión. Más concretamente, el ámbito de aplicación del Reglamento son los biocidas que, en la forma en que se suministran al usuario, estén compuestos por una o más sustancias activas, o bien que las generen. Asimismo, se distingue entre sustancias activas existentes, que ya estaban introducidas en el mercado en la fecha de transposición fijada en la Directiva 98/8/CE, y sustancias activas nuevas o que no estaban introducidas en el mercado en dicha fecha. 
Para garantizar el principio de seguridad jurídica, el Reglamento contiene una lista de sustancias activas cuyo uso como ingredientes de biocidas ha sido aprobado previamente. Ahora bien, se establece un procedimiento para evaluar si una sustancia activa puede o no incluirse en esa lista. Así, las sustancias activas con los peores perfiles de peligro no deben aprobarse para su uso en biocidas, salvo en situaciones específicas, como aquellas en las que la aprobación se justifique por ser desdeñable el riesgo de exposición a la sustancia, por motivos relacionados con la salud humana o animal o el medio ambiente, o por no entrañar efectos negativos desproporcionados para la sociedad.

Por último, el Reglamento establece la prohibición de comercializar y usar biocidas que no hayan sido autorizados de conformidad con la norma comunitaria. Pues bien, también se dispone que durante el procedimiento de concesión o de renovación de la autorización de un biocida que contenga una sustancia activa candidata a sustitución, es importante que se pueda comparar el biocida con otros biocidas autorizados y con métodos de control y prevención que no sean químicos en función de los riesgos que plantean y los beneficios de su uso. A raíz de tal evaluación comparada, un biocida que contenga sustancias activas calificadas como candidatas a sustitución debe quedar prohibido o sometido a restricciones si se demuestra que hay otros biocidas autorizados $\mathrm{u}$ otros métodos de control o prevención que no sean químicos que presenten un riesgo global significativamente menor para la salud humana y animal y para el medio ambiente, que sean suficientemente eficaces y que no presenten otros inconvenientes económicos o prácticos de consideración. En tales casos, deberán establecerse plazos de retirada progresiva.

La segunda norma que debemos destacar es el Reglamento 500/2012, de 13 de junio de 2012, que modifica el Reglamento (CE) 302/2009 del Consejo por el que se establece un plan de recuperación plurianual para el atún rojo del Atlántico oriental y el Mediterráneo. La Unión es parte del Convenio Internacional para la Conservación del Atún del Atlántico, en cuya 17. a reunión especial en 2010 se adoptó la Recomendación 10-04, que modifica el plan de recuperación plurianual para el atún rojo.

Con el objeto de reconstituir la población de esta especie, la Recomendación 10-04 prevé una nueva reducción del total admisible de capturas y refuerza tanto las medidas dirigidas a disminuir la capacidad pesquera como las medidas de control, especialmente en lo relativo a las transferencias y las operaciones de introducción en jaula. Asimismo, 
prevé el asesoramiento adicional del Comité Permanente de Investigación y Estadísticas en 2012 con respecto a la identificación de las zonas de desove y la creación de santuarios.

A la vista de la Recomendación, algunas disposiciones de los reglamentos europeos quedaron lógicamente desfasadas, de ahí que se proceda ahora a su eliminación o modificación. Asimismo, para prever unas condiciones uniformes en lo que se refiere a las operaciones de transferencia, las operaciones de introducción en jaula y el registro y la notificación de las actividades de las almadrabas de túnidos, se otorgan a la Comisión competencias de ejecución. Dichas competencias serán ejercidas de conformidad con el Reglamento 182/2011 del Parlamento Europeo y del Consejo, de 16 de febrero de 2011, por el que se establecen las normas y los principios generales relativos a las modalidades de control por parte de los Estados miembros del ejercicio de las competencias de ejecución por la Comisión.

Por último, debemos dar noticia de la Directiva 2012/19/UE del Parlamento y del Consejo, de 4 de julio de 2012, que refunde las directivas sobre residuos de aparatos eléctricos y electrónicos (RAEE). La Directiva pretende dar solución a un problema ambiental relacionado con la expansión de los mercados y la reducción de los ciclos de innovación, así como con la aceleración de la sustitución de los aparatos eléctricos y electrónicos, que convierte rápidamente los AEE en una creciente fuente de residuos.

Desde este punto de vista, la Directiva completa la normativa general de la Unión sobre gestión de residuos, como la Directiva 2008/98/CE del Parlamento Europeo y del Consejo, de 19 de noviembre de 2008, sobre los residuos, y modifica la Directiva 2002/96/CE del Parlamento Europeo y del Consejo, de 27 de enero de 2003, sobre residuos de aparatos eléctricos y electrónicos (RAEE), en aras de una mayor claridad.

Concretamente, la nueva Directiva tiene por objetivo contribuir a la producción y el consumo sostenibles mediante, de forma prioritaria, la prevención de la generación de RAEE y, además, la reutilización, el reciclado y otras formas de valorización de dichos residuos, a fin de reducir su eliminación y contribuir al uso eficaz de los recursos y a la recuperación de materias primas secundarias valiosas. Asimismo, pretende mejorar el comportamiento medioambiental de todos los agentes que intervienen en el ciclo de vida de los AEE como, por ejemplo, los productores, distribuidores y consumidores, $\mathrm{y}$, en particular, de aquellos agentes directamente implicados en la recogida y el tratamiento de los RAEE. 
Relacionado con lo anterior, la Directiva pone de relieve que la distinta aplicación nacional del principio de responsabilidad del productor puede hacer que los agentes económicos soporten cargas financieras muy desiguales. Del mismo modo, la existencia de políticas nacionales dispares en materia de gestión de los RAEE reduce la eficacia de las políticas de reciclado. Por ese motivo, se establecen criterios fundamentales a nivel de toda la Unión con normas mínimas relativas al tratamiento de los RAEE.

Una consideración importante es que las disposiciones de la Directiva deberán aplicarse a productos y productores con independencia de la técnica de venta empleada, inclusive la venta a distancia y la venta electrónica. En ese sentido, las obligaciones de productores y distribuidores que utilizan canales de venta a distancia y electrónicos deben adoptar, en la medida de lo posible, la misma forma y deben aplicarse de la misma manera que en otros canales de distribución, con objeto de evitar que esos otros canales de distribución tengan que soportar los costes derivados de las disposiciones de la Directiva en lo que se refiere a los RAEE de equipos vendidos mediante venta a distancia o electrónica. Igualmente, el ámbito de aplicación de la Directiva comprende todos los AEE, tanto los de consumo como los de uso profesional.

La Directiva también establece que la responsabilidad del productor es uno de los medios para estimular el diseño y producción de AEE. Asimismo, el productor debe facilitar su reparación y su posible actualización, así como su reutilización, desmontaje y reciclado. Pues bien, la recogida separada es condición previa para asegurar el tratamiento y reciclado específicos de los RAEE. Para ello, asimismo, se promueve que los consumidores contribuyan activamente al éxito de dicha recogida.

Por otro lado, con el fin de alcanzar el nivel deseado de protección, la Directiva intima a los Estados miembros a tomar las medidas adecuadas para reducir al mínimo la eliminación de RAEE como residuos urbanos no seleccionados, logrando un alto grado de recogida separada. Por este motivo, se prevé que los usuarios de AEE de hogares particulares puedan tener la posibilidad de devolver sus RAEE al menos sin cargo alguno. Para ello, los productores deberán financiar al menos la recogida en las instalaciones de recogida, así como el tratamiento, la valorización y la eliminación de los RAEE. En esta dirección, se prevé que los Estados fomenten que los productores asuman plenamente la recogida de los RAEE, en particular, financiando la recogida a lo largo de toda la cadena de residuos, incluso los procedentes de hogares particulares, con el fin de evitar que los RAEE recogidos de modo separado sean objeto de tratamiento 
inadecuado y de exportación ilegal; además, todo ello contribuye a que el pago por la recogida de estos residuos corresponda a los consumidores, de acuerdo con el principio "quien contamina paga", y no al contribuyente en general.

\subsection{Actos del Consejo}

\section{A. Reglamentos}

El Reglamento 692/2012, de 24 de julio de 2012, por el que se modifican los reglamentos (UE) 43/2012 y (UE) 44/2012 en lo que atañe a la protección de la Manta birostris y a determinadas posibilidades de pesca, es el resultado de la décima Conferencia de las Partes (COP 10) de la Convención sobre la Conservación de las Especies Migratorias de Animales Silvestres, celebrada en Bergen del 20 al 25 de noviembre de 2011, en la que se añadió la manta (Manta birostris) a la lista de especies protegidas de los apéndices I y II de la Convención. Pues bien, mediante este reglamento se procede a proteger las poblaciones de manta en todas las aguas en lo que respecta a los buques que pesquen en aguas de la UE, ya sean buques de la UE, ya buques de terceros países.

\section{B. Decisiones}

En dos decisiones el Consejo advierte de la necesidad de la incorporación al Acuerdo Internacional EEE de los nuevos actos dictados por las instituciones europeas en materia medioambiental y al mismo tiempo se toma la posición común a mantener en el marco del Comité Mixto EEE. Se trata de las decisiones del Consejo, de 10 de julio de 2012 y de 24 de julio de 2012, relativas a la posición que debe adoptar el Comité Mixto EEE en relación con la modificación del anexo XX (medio ambiente) del Acuerdo EEE sobre la incorporación al Acuerdo, por una parte, del Reglamento 268/2010, el Reglamento 1088/2010, el Reglamento 1089/2010 y el Reglamento 102/2011, y, por otra, de la Directiva 2009/29/CE sobre las emisiones de gases de efecto invernadero, del Reglamento 1031/2010 en relación con las subastas de los derechos de emisión de estos gases o de la Directiva 2003/87/CE, entre otros.

Por otro lado, se ha adoptado la Decisión del Consejo, de 10 de julio de 2012, por la que se establece la posición que debe adoptarse en nombre de la Unión Europea en relación 
con las propuestas de enmienda a los anexos II y III del Protocolo sobre las zonas especialmente protegidas y la diversidad biológica en el Mediterráneo del Convenio para la Protección del Medio Marino y de la Región Costera del Mediterráneo en la decimoséptima reunión de las Partes contratantes (París, Francia, 8-10 de febrero de 2012). La Decisión realiza una enmienda a los anexos II y III del citado Protocolo donde se enumeran, respectivamente, las especies en peligro o amenazadas y las especies cuya explotación está regulada. La modificación tiene por objeto trasladar diez especies de tiburones del anexo III al anexo II con el fin de otorgar a estas especies una mayor protección.

\subsection{Actos de la Comisión}

A. Reglamentos de la Comisión y reglamentos de ejecución

El Reglamento 493/2012, de 11 de junio de 2012, establece, de conformidad con la Directiva 2006/66/CE del Parlamento Europeo y del Consejo, normas detalladas para el cálculo de los niveles de eficiencia de los procesos de reciclado de los residuos de pilas y acumuladores. El Reglamento define el proceso de reciclado como un proceso que empieza tras la recogida y eventual clasificación y/o preparación para el reciclado de los residuos de pilas y acumuladores recibidos por una instalación de reciclado, y termina cuando se producen fracciones de salida que van a utilizarse para los mismos fines a los que se destinaban originalmente o para otros fines sin ningún otro tratamiento y que han dejado de ser residuos. Pues bien, para fomentar el desarrollo de nuevas tecnologías de reciclado y tratamiento y la mejora de las existentes, se prevé que cada uno de los procesos de reciclado deberá alcanzar determinados niveles de eficiencia de reciclado. A tal efecto, se establece un plazo mínimo de dieciocho meses para que las empresas de reciclado de residuos de pilas y acumuladores adapten sus procesos tecnológicos a los nuevos requisitos en materia de cálculo de los niveles de eficiencia de reciclado.

El segundo acto jurídico a destacar es el Reglamento 601/2012, de 21 de junio de 2012, sobre el seguimiento y la notificación de las emisiones de gases de efecto invernadero en aplicación de la Directiva 2003/87/CE del Parlamento Europeo y del Consejo. El seguimiento y la notificación de las emisiones de gases de efecto invernadero de una manera exhaustiva, coherente, transparente y exacta de acuerdo con los requisitos armonizados establecidos en este reglamento son fundamentales para que funcione 
eficazmente el régimen de comercio de derechos de emisión de gases de efecto invernadero previsto en la Directiva 2003/87/CE.

Para optimizar el funcionamiento del sistema de seguimiento y notificación, se prevé que los Estados miembros que designen a más de una autoridad competente deberán velar porque estas coordinen su trabajo de acuerdo con los principios establecidos en el Reglamento en sus artículos 4 a 10, esto es, sobre la base de los principios de exhaustividad, coherencia, comparabilidad y transparencia, exactitud, integridad de la metodología, mejora continua y coordinación.

El núcleo del sistema establecido por el Reglamento afecta a las actividades de aviación relacionadas en el anexo I de la Directiva 2003/87/CE. En los artículos 11 a 17 del Reglamento se prevé el plan de seguimiento, que debe ser aprobado con documentación pormenorizada, completa y clara de la metodología seguida por cada titular de instalación u operador de aeronaves. Dicho plan debe ser objeto de actualizaciones periódicas, tanto en respuesta a los resultados de los verificadores como por propia iniciativa del titular de instalación u operador de aeronaves.

Por último, para cerrar posibles lagunas en materia de transferencias de $\mathrm{CO}_{2}$ inherente o puro, se prevé que solo deberán permitirse esas transferencias en condiciones muy específicas. Tales condiciones consisten, en concreto, en que la transferencia de $\mathrm{CO}_{2}$ inherente se haga exclusivamente a otras instalaciones acogidas al régimen de comercio de derechos de emisión de la Unión (RCDE UE), y que la transferencia de $\mathrm{CO}_{2}$ inherente se haga exclusivamente para fines de almacenamiento geológico en un emplazamiento autorizado por el RCDE UE, única modalidad de almacenamiento permanente de $\mathrm{CO}_{2}$ admitida por dicho régimen.

El Reglamento 519/2012, de 19 de junio de 2012, por el que se modifica el Reglamento (CE) 850/2004 del Parlamento Europeo y del Consejo, sobre contaminantes orgánicos persistentes, con respecto al anexo I, incorpora a la legislación de la Unión los compromisos establecidos en el Convenio de Estocolmo sobre contaminantes orgánicos persistentes. A la vista de las decisiones adoptadas en el marco del Convenio de 1979 sobre la contaminación atmosférica transfronteriza a gran distancia provocada por contaminantes orgánicos persistentes (en lo sucesivo, "el CLRTAP"), se ha puesto de relieve la necesidad de actualizar el anexo I, parte B, del Reglamento 850/2004. 
La comercialización y el uso de las PCCC (y las parafinas cloradas de cadena corta) en la Unión se referían hasta ahora solamente a dos usos, ámbito este mucho más limitado que el previsto de acuerdo con las previsiones del CLRTAP. Así pues, el Reglamento procede a ampliar el ámbito de la restricción aplicable a las PCCC prohibiendo su producción, comercialización y utilización, excepto en relación con los usos exentos previstos. Igualmente, se modifican las excepciones previstas a este respecto.

El siguiente acto jurídico aprobado por la Comisión es el Reglamento 791/2012, de 23 de agosto de 2012, que modifica, en lo relativo a ciertos requisitos sobre el comercio de especies amenazadas de fauna y flora silvestres, el Reglamento (CE) 865/2006, por el que se establecen disposiciones de aplicación del Reglamento (CE) 338/97 del Consejo. Con objeto de aplicar determinadas resoluciones adoptadas en la decimoquinta reunión de la Convención sobre el Comercio Internacional de Especies Amenazadas de Fauna y Flora Silvestres (CI-TES), se procede a modificar algunas previsiones del Reglamento 865/2006 de la Comisión, de 4 de mayo de 2006, por el que se establecen disposiciones de aplicación del Reglamento 338/97 del Consejo, relativo a la protección de especies de la fauna y flora silvestres mediante el control de su comercio, así como a añadir otras nuevas. En concreto, se modifican las disposiciones relativas a las condiciones que rigen la identificación y el marcado de especímenes, a la emisión de algunos documentos con carácter retroactivo, a las condiciones en las que pueden emitirse certificados de propiedad privada, al régimen aplicable en la Unión a los efectos personales y enseres domésticos y a su reexportación, a las condiciones en las que los especímenes del anexo A pueden ser objeto de actividades comerciales en la Unión, y a las condiciones que se aplican a los certificados emitidos anticipadamente.

También ha sido aprobado el Reglamento de Ejecución 274/2012, de 27 de marzo de 2012, que modifica el Reglamento (CE) 1152/2009, por el que se establecen condiciones específicas para la importación de determinados productos alimenticios de algunos terceros países debido al riesgo de contaminación de dichos productos por aflatoxinas. En la medida en que ha cambiado el código de la nomenclatura combinada (NC) de determinadas categorías de productos alimenticios contempladas en el Reglamento 1152/2009, procede modificar los códigos NC de dicho Reglamento. Asimismo, se derogan las disposiciones transitorias que afectan a los productos alimenticios importados de los Estados Unidos que no estén incluidos en el Voluntary Aflatoxin Sampling Plan ("Plan Voluntario de Muestreo de Aflatoxinas"), dado que se 
ha concedido un plazo suficiente a los explotadores de los Estados Unidos para que apliquen dicho plan.

Por otro lado, en virtud del número y la naturaleza de las notificaciones del sistema de alerta rápida para los piensos y los alimentos, del volumen comercial, del resultado de las inspecciones de la Oficina Alimentaria y Veterinaria y del resultado de los controles, se reducen en algunos casos la frecuencia del muestreo para análisis. Es el caso de las avellanas procedentes de Turquía y de las nueces procedentes de Brasil.

Relacionado temáticamente con el anterior reglamento, ha sido dictado también el Reglamento de Ejecución 792/2012, de 23 de agosto de 2012, por el que se establecen disposiciones sobre el diseño de los permisos, certificados y otros documentos previstos en el Reglamento (CE) 338/97 del Consejo, relativo a la protección de especies de la fauna y flora silvestres mediante el control de su comercio, y se modifica el Reglamento (CE) 865/2006 de la Comisión. Para asegurar una aplicación uniforme del Reglamento (CE) 338/97, se establecen los modelos a los que deberán ajustarse los permisos, certificados y demás documentos previstos en los reglamentos. Esas condiciones uniformes son adoptadas de acuerdo con el procedimiento de examen previsto en el artículo 5 del Reglamento (UE) 182/2011 del Parlamento Europeo y del Consejo, de 16 de febrero de 2011, por el que se establecen las normas y los principios generales relativos a las modalidades de control por parte de los Estados miembros del ejercicio de las competencias de ejecución de la Comisión.

Para concluir con la labor normativa de la Comisión, han sido aprobados dos grupos de reglamentos — reglamentos de la Comisión y reglamentos de ejecución-, relativos a la prohibición de pesca de distintas especies. En cuanto a los reglamentos de la Comisión, cabe citar los siguientes:

- Reglamento 916/2012, de 2 de octubre de 2012, por el que se prohíbe la pesca de gallineta nórdica en la zona NAFO $3 \mathrm{M}$ por parte de los buques que enarbolan pabellón de Lituania.

- Reglamento 915/2012, de 28 de septiembre de 2012, por el que se prohíbe la pesca de anchoa en las zonas IX y X; aguas de la UE de CPACO 34.1.1 por parte de los buques que enarbolan pabellón de España. 
- Reglamento 914/2012, de 28 de septiembre de 2012, por el que se prohíbe la pesca de gallineta nórdica en la zona NAFO $3 \mathrm{M}$ por parte de los buques que enarbolan pabellón de España.

- Reglamento 913/2012, de 28 de septiembre de 2012, por el que se prohíbe la pesca de solla en las zonas VIIf y VIIg por parte de los buques que enarbolan pabellón de Bélgica.

- Reglamento 912/2012, de 28 de septiembre de 2012, por el que se prohíbe la pesca de gallineta nórdica en la zona NAFO $3 \mathrm{M}$ por parte de los buques que enarbolan pabellón de Letonia.

- Reglamento 911/2012, de 28 de septiembre de 2012, por el que se prohíbe la pesca de gallineta nórdica en la zona NAFO $3 \mathrm{M}$ por parte de los buques que enarbolan pabellón de Estonia.

— Reglamento 910/2012, de 28 de septiembre de 2012, por el que se prohíbe la pesca de bacalao en Skagerrak por parte de los buques que enarbolan pabellón de Suecia.

- Reglamento 909/2012, de 28 de septiembre de 2012, por el que se prohíbe la pesca del lenguado común en la zona de IIIa; aguas de la UE de las subdivisiones 22-23, por parte de los buques que enarbolan pabellón de Suecia.

- Reglamento 888/2012, de 25 de septiembre de 2012, por el que se prohíbe la pesca de eglefino en las zonas VIIb-k, VIII, IX y X y en aguas de la UE del CPACO 34.1.1 por parte de los buques que enarbolan pabellón de Bélgica.

- Reglamento 887/2012, de 24 de septiembre de 2012, por el que se prohíbe la pesca de gallineta nórdica en la zona NAFO $3 \mathrm{M}$ por parte de los buques que enarbolan pabellón de Alemania.

- Reglamento 886/2012, de 21 de septiembre de 2012, por el que se prohíbe la pesca de gallos en aguas de la UE de las zonas de IIa y IV por parte de los buques que enarbolan pabellón de Dinamarca.

- Reglamento 882/2012, de 21 de septiembre de 2012, por el que se prohíbe la pesca de bacalao en la zona IV, en aguas de la UE de la zona IIa, y en la parte de la zona IIIa no incluida en el Skagerrak y el Kattegat por parte de los buques que enarbolan pabellón de Suecia. 
- Reglamento 883/2012, de 21 de septiembre de 2012, por el que se prohíbe la pesca de bacalao en aguas de Noruega de las zonas I y II por parte de los buques que enarbolan pabellón de Portugal.

- Reglamento 884/2012, de 21 de septiembre de 2012, por el que se prohíbe la pesca de eglefino en aguas de Noruega de las zonas I y II por parte de los buques que enarbolan pabellón de Portugal.

- Reglamento 881/2012, de 21 de septiembre de 2012, por el que se prohíbe la pesca de gallineta nórdica en la zona NAFO $3 \mathrm{M}$ por parte de los buques que enarbolan pabellón de Portugal.

- Reglamento 775/2012, de 23 de agosto de 2012, por el que se prohíbe la pesca de sable negro en aguas de la UE y aguas internacionales de las zonas VIII, IX y X por parte de los buques que enarbolan pabellón de España.

- Reglamento 770/2012, de 21 de agosto de 2012, por el que se prohíbe la pesca de eglefino en aguas de la UE y aguas internacionales de las zonas Vb y VIa por parte de los buques que enarbolan pabellón de España.

- Reglamento 769/2012, de 17 de agosto de 2012, por el que se prohíbe la pesca de alfonsino en aguas de la UE y aguas internacionales de las zonas III, IV, V, VI, VII, VIII, IX, X, XII, XIV por parte de los buques que enarbolan pabellón de Portugal.

- Reglamento 768/2012, de 17 de agosto de 2012, por el que se prohíbe la pesca de brótola en aguas de la UE y aguas internacionales de las zonas VIII y IX por parte de los buques que enarbolan pabellón de Portugal.

Finalmente, y en relación con los reglamentos de ejecución dictados en esta materia, se han de mencionar los siguientes:

— Reglamento de Ejecución 920/2012, de 4 de octubre de 2012, por el que se prohíben las actividades pesqueras de los palangreros que enarbolan pabellón de Chipre o están matriculados en Chipre y practican la pesca de atún rojo en el océano Atlántico, al este del meridiano $45^{\circ} \mathrm{O}$, y en el mar Mediterráneo.

- Reglamento de Ejecución 767/2012, de 17 de agosto de 2012, por el que se prohíbe la pesca de atún rojo en el océano Atlántico, al este del meridiano $45^{\circ} \mathrm{O}$, y en el mar Mediterráneo por parte de los buques que enarbolan pabellón de Portugal. 
— Reglamento de Ejecución 721/2012, de 4 de julio de 2012, por el que se prohíben las actividades pesqueras de las almadrabas y palangreros con pabellón o matrícula de Grecia o Malta que practican la pesca de atún rojo en el océano Atlántico al este del meridiano $45^{\circ} \mathrm{O}$ y en el mar Mediterráneo.

— Reglamento de Ejecución 606/2012, de 4 de julio de 2012, por el que se prohíben las actividades pesqueras con almadrabas y palangreros con pabellón o matrícula de Italia que practican la pesca de atún rojo en el océano Atlántico, al este del meridiano $45^{\circ} \mathrm{O}$, y en el mar Mediterráneo.

- Reglamento de Ejecución 605/2012, de 4 de julio de 2012, por el que se prohíben las actividades pesqueras de las almadrabas registradas en España que practican la pesca de atún rojo en el océano Atlántico, al este del meridiano $45^{\circ} \mathrm{O}$, y en el mar Mediterráneo.

\section{B. Decisión de ejecución}

En el marco de la protección de especies piscícolas, la Decisión de Ejecución de la Comisión, de 16 de mayo de 2012, que modifica la Decisión 2008/589/CE por la que se establece un programa específico de control e inspección de las poblaciones de bacalao del mar Báltico (2012/262/UE), ha sido dictada para resolver la cuestión de la posible notificación incorrecta en las pesquerías que explotan las poblaciones de salmón del mar Báltico.

Por su parte, la Decisión de Ejecución de la Comisión, de 7 de mayo de 2012, relativa a la determinación de los períodos de arranque y de parada a efectos de la Directiva 2010/75/UE del Parlamento Europeo y del Consejo, sobre las emisiones industriales [notificada con el número C(2012) 2948] (2012/249/UE), ha sido dictada porque la Directiva 2010/75/UE no determinaba los períodos de arranque (o puesta en marcha) y de parada, a pesar de que varias disposiciones de la citada Directiva hacían referencia a dichos períodos.

\section{Dictámenes}

El Dictamen de la Comisión, de 30 de marzo de 2012, relativo al plan de evacuación de los residuos radiactivos resultantes de la ampliación del depósito de residuos de muy baja actividad CSTFA, situado en Francia, de conformidad con el artículo 37 del 
Tratado Euratom (2012/C99/01), señala que la ampliación de este depósito no supondrá en ningún caso una contaminación radiactiva del agua, del suelo o del espacio aéreo. Aunque se entiende que del funcionamiento normal de las instalaciones se desprenderán gases a la atmósfera, estos no serán significativos desde el punto de vista sanitario y medioambiental.

Finalmente, tanto el Dictamen de la Comisión, de 30 de mayo de 2012, relativo al plan de evacuación de residuos radiactivos procedentes de las instalaciones de almacenamiento provisional de residuos de actividad media y combustible gastado de la central nuclear de Hinkley Point C, situada en Somerset (Reino Unido), de conformidad con el artículo 37 del Tratado Euratom (2012/C151/01), como el Dictamen de la Comisión, de 11 de junio de 2012, relativo al plan de evacuación de los residuos radiactivos resultantes de ITER (Reactor Termonuclear Experimental Internacional), situado en Cadarache, Francia, de conformidad con el artículo 37 del Tratado Euratom (2012/C 166/01), afirman, respectivamente, que el traslado de los mencionados residuos no supondrá en ningún caso una contaminación radiactiva líquida o gaseosa del agua, del suelo o del espacio aéreo. Asimismo, en ambos casos se afirma que en caso de accidente las dosis recibidas por la población y el medio ambiente no serán significativas desde el punto de vista sanitario y medioambiental.

\subsection{Actos del Parlamento}

El Parlamento ha aprobado varias resoluciones y declaraciones en materia ambiental. La primera de ellas es la Resolución de 25 de noviembre de 2010, sobre los derechos humanos y las normas sociales y medioambientales en los acuerdos comerciales internacionales (2009/2219(INI)) (2012/C 99 E/07). El Parlamento Europeo, en esta resolución, parte de la base de que el vínculo entre comercio, derechos humanos y normas sociales y medioambientales se ha convertido en un elemento clave de las relaciones económicas y comerciales y es parte integrante de las negociaciones en el marco de los acuerdos de libre comercio. Destaca, asimismo, que el sector del comercio y el respeto de las normas relativas a los derechos humanos, así como las relacionadas con los ámbitos social y medioambiental, son aspectos importantes para garantizar la paz y el bienestar en el mundo, pero que no pueden considerarse la solución a todos los problemas que puedan surgir entre los distintos Estados del mundo. Se considera, no obstante, que se puede superar el estancamiento en la situación política mediante el 
fortalecimiento de las relaciones comerciales, garantizando así la definición de intereses comunes, sobre todo en el ámbito de la protección medioambiental, como forma de dirimir conflictos.

El Parlamento Europeo solicita, en virtud de estos argumentos, que, en el marco de la futura estrategia comercial de la Unión Europea, el comercio no sea considerado como un fin en sí mismo, sino como una herramienta que permita la promoción de los valores y de los intereses comerciales europeos, así como un instrumento para lograr unos intercambios más justos, capaces de generalizar la inclusión y la aplicación efectivas de normas sociales y medioambientales con respecto a todos los socios comerciales de la UE. Por todo ello, el Parlamento opina que la Unión Europea debería guiarse por un enfoque positivo $\mathrm{y}$, al mismo tiempo, jurídicamente vinculante durante sus negociaciones, subrayando que la inclusión de disposiciones relativas al desarrollo sostenible, en particular en los acuerdos bilaterales, beneficiará a todas las partes interesadas.

Por su parte, la Declaración de 16 de diciembre de 2010, sobre el apoyo al endurecimiento de la prohibición del cercenamiento de aletas de tiburón en la UE (2012/C169E/18), insta a la Comisión a crear una propuesta para la prohibición del cercenamiento de aletas de tiburón en la Unión, debido a que la mayor parte de la población de tiburones en la Unión Europea está en peligro de extinción y la práctica del cercenamiento, que supone seccionar la aleta del animal y tirar el resto al mar, está dando lugar a una agresiva disminución de las especies en territorio europeo.

\subsection{Otros}

El Comité de las Regiones ha aprobado un interesante dictamen titulado "Ciudades del mañana: ciudades sostenibles desde el punto de vista social y medioambiental" (2012/C277/04). Este dictamen se basa en su activa participación en la $5^{\text {a }}$ Cumbre de las Regiones y Ciudades celebrada los días 22 y 23 de marzo de 2012 en Copenhague en el marco de la estrategia Europa 2020.

El Dictamen versa sobre el diseño del modelo territorial en relación con los conflictos sociales y medioambientales. Desde una perspectiva estrictamente medioambiental, el Comité apoya la promoción de estrategias integrales para un desarrollo urbano sostenible, la cooperación horizontal y vertical en un sistema de gobernanza 
participativa que tenga en cuenta la diversidad de las ciudades y la importancia de la innovación social, y una planificación previsora.

Asimismo, subraya los retos que plantea el cambio climático y la necesidad de reducir el consumo de energía por medio de procesos de recuperación de la energía y de aumento de la eficiencia energética, de crear sistemas en red, de ampliar los espacios libres y verdes, así como de introducir nuevas formas de construcción y formas de movilidad sanas y seguras que no malgasten recursos.

Todas estas recomendaciones van dirigidas a las instituciones europeas, pero también se invita a los Estados miembros a que reactiven sus esfuerzos en materia de política urbana e incluyan igualmente en sus iniciativas los ámbitos no relacionados con la política territorial, resaltando especialmente la importancia de la cooperación entre ciudades y aglomeraciones urbanas como indispensable para garantizar un desarrollo sostenible.

También el Comité de las Regiones ha aprobado el Dictamen relativo a la "Directiva sobre el ruido ambiental: El camino a seguir" (2012/C 113/08). En este dictamen, aunque se reconoce que la contaminación acústica es fundamentalmente un problema local, se afirma que requiere una solución a nivel europeo y, por ello, se solicita la elaboración de una ambiciosa política europea en materia de ruido en forma de medidas que ataquen el problema en la fuente. En este sentido, el Dictamen admite la importancia y la repercusión de la Directiva sobre el ruido ambiental y se congratula de los beneficios reales aportados por ella. Sin embargo, considera necesario que se amplíe su ámbito de aplicación, haciendo referencia específica a las entidades locales y regionales, dado que desempeñan un papel esencial en la lucha contra el ruido excesivo.

El Comité propone a la Comisión que, tras una minuciosa evaluación de sus repercusiones para los entes locales y regionales, establezca valores umbral de ruido y objetivos basándose en las recomendaciones de la OMS en materia de salud. Por otro lado, reitera la necesidad de articular entre sí y complementar los diversos instrumentos normativos que regulan los materiales relacionados con las fuentes de ruido y de resolver las lagunas normativas existentes (especialmente en lo que respecta a los vehículos y, en particular, a los automóviles y camiones, carreteras, ferrocarriles y aeropuertos) estableciendo un marco jurídico con un tronco común. 
Otra de las recomendaciones a la Comisión es que se amplíe el concepto de la gobernanza multinivel a otros ámbitos, tales como el ruido, utilizando como referencia el Pacto de los Alcaldes.

Para concluir, también el Comité Económico y Social Europeo (CESE) ha emitido varios dictámenes en materia ambiental que merece la pena destacar. El primero de ellos es el Dictamen sobre la "Comunicación de la Comisión al Parlamento Europeo, al Consejo y al Comité Económico y Social Europeo relativa a la estrategia de la Unión Europea para la protección y el bienestar de los animales 2012-2015" [COM(2012) 6 final], (2012/C 229/20). El CESE comparte de modo general la estrategia sobre bienestar animal presentada por la Comisión, apoyando la legítima aspiración de los consumidores a la salud alimentaria; apoya, además, una estrategia productiva europea orientada hacia la calidad. Sin embargo, el CESE encuentra que hay problemas en la implantación de las normas existentes derivados de la falta de apoyo a su aplicación y de la pérdida de competitividad de las producciones comunitarias. En este sentido, y puesto que faltan instrumentos para compensar la pérdida de competitividad de las producciones ganaderas comunitarias, y en la medida en que no todos los productos importados cumplen los mismos estándares de producción impuestos a las producciones comunitarias, surgen así desigualdades insostenibles. El CESE llama la atención, a estos efectos, sobre la importancia de destinar una parte de los fondos de cooperación a la formación de autoridades, empresarios y trabajadores de países terceros en materia de bienestar en la producción animal.

Se señala, igualmente, que debe diseñarse una estrategia de comunicación enfocada hacia las preocupaciones de la sociedad, teniendo en cuenta los estudios y avances científicos en la materia y los diferentes puntos de vista de productores, trabajadores y consumidores. El CESE opina, finalmente, que la Red de Centros Europeos de Referencia debe contar con la participación de todos los agentes sociales y de los consumidores, y que tiene un papel crucial en el desarrollo de la estrategia de bienestar animal.

Para terminar, se debe hacer alusión al Dictamen sobre "La posición del CESE para la preparación de la Conferencia de las Naciones Unidas sobre Desarrollo Sostenible (Río+20)" (Dictamen adicional) (2012/C143/08). En virtud de los estudios realizados por el CESE en esta materia, se indica la necesidad de un cambio en el enfoque de las políticas europeas. En este sentido, se propone que en la Conferencia se adopte un plan 
de acción concreto que conduzca a un desarrollo más sostenible, fomentando una economía verde que establezca un equilibrio entre los aspectos sociales, económicos y ecológicos para alcanzar una equidad distributiva e intergeneracional.

Centrándonos en el estricto ámbito medioambiental, el CESE establece que es necesario adoptar una economía ecológica a través de distintas políticas. Por una parte, los Estados deberían adquirir más conciencia de la limitación de nuestros recursos naturales y reducir su consumo, utilizando los mínimos recursos naturales de manera más eficiente. Por otra, el CESE recomienda realizar un plan que sustituya el consumo y la producción insostenibles en un plazo de diez años. Igualmente, se aboga por un acuerdo mundial entre países para adoptar estos cambios y que sean significativos, así como la adhesión a estas políticas de las entidades y empresas privadas.

Para efectuar todos estos cambios, el CESE recomienda crear un nuevo Consejo para el Desarrollo Sostenible que sustituya a la antigua Comisión. Estima además que es necesario que ese Consejo dé cabida a la representación o participación de la sociedad civil. Del mismo modo, considera acertada la creación de la figura del Defensor de las generaciones futuras. 